Message for others Based on current evidence, we believe that the ICR model should be taken into consideration in the future design and planning of any neonatal unit.

\section{G573(P) IMPROVING EASE AND ACCURACY OF CENTILE CALCULATIONS WITH A SMARTPHONE IMPLEMENTATION OF RCPCH UK-WHO GROWTH CHARTS}

CJ Kelly. Centre for the Developing Brain, King's College London, London, UK

10.1136/archdischild-2015-308599.522

Context Improvement work performed while working as a paediatric trainee ST2 on a tertiary neonatal intensive care unit, neurosurgical ward and academic research department. Target audience: health professionals who calculate growth centiles on a regular basis.

Problem Calculating growth centiles and their trends are a crucial part of the general paediatric and neonatal assessment. However, due to the regular scarcity of paper charts and inconvenience of trying to find one on a busy ward, centiles are often overlooked. Even with a chart available for use, the manual plotting method gives broad estimates rather than exact numbers (i.e. $25-50^{\text {th }}$ centile), which are not appropriate for research purposes.

Assessment of problem and analysis of its causes Working on a paediatric neurosurgical ward, the neurosurgical consultants complained that growth centiles were not being filled in consistently by junior doctors during clerking. A poll of fellow trainees agreed that an electronic version, available at the point of care, would be very beneficial. Problems with paper growth charts include lack of chart availability, time pressure, plotting inaccuracies, and inherent centile approximation due to threshold banding. Additionally, the calculation of corrected age for children and post menstrual ages for premature babies is commonly performed inaccurately.

A solution to this problem would need to combine improved accuracy with greater ease of calculation, providing a tool that staff could carry with them everywhere at work.

Intervention Creation of a smartphone app to calculate growth centiles for premature babies through to 18 year old children, using RCPCH UK-WHO data. The app also addresses the difficulty of calculating corrected ages by building this into the calculation.

Strategy for change The provisional app was circulated to a number of interested paediatricians, and very helpful feedback received from the NHS Hackday group. The app was also discussed with Prof Tim Cole (the creator of the original growth charts), who provided some very helpful feedback and suggestions for improvement.

The app was launched as a free download in October 2014 on iPhone and Android as a proof of concept via social media, and a significant amount of constructive feedback was received. An updated version is currently in progress, building in many of these suggestions.

Measurement of improvement The project is at an early stage, and no objective metrics of improvement have yet been collected. It is hoped that this will be possible after the launch of the final version.

\section{GrowthCharts $^{\text {ukmomo }}$}

\section{Current gestational age: $40+2$ weeks, day 7 today*}

\section{RCPCH UK-WHO Growth Chart for Boys}

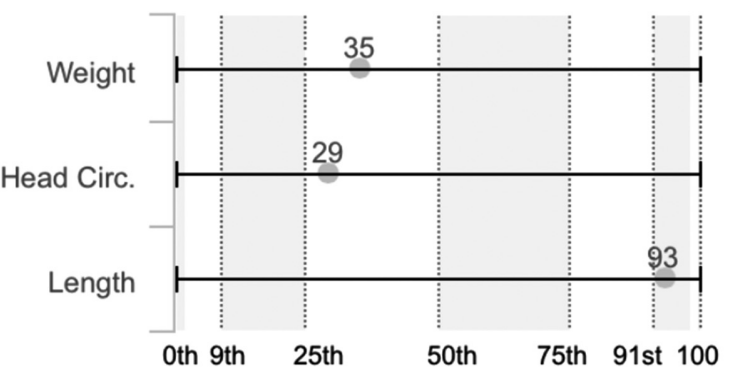

\section{Weight $3.5 \mathrm{~kg} 25$ th - 50th centile (35th) \\ Head $35 \mathrm{~cm} 25$ th -50 th centile (29th) \\ Length $55 \mathrm{~cm} 91 \mathrm{st}$ - 98th centile (93rd)}

\section{Measured on Sun 7th Sep 2014}

\section{Modify Details}

\section{New Chart}

\section{Source information and Disclaimer}

Abstract G573(P) Figure 1

Effects of changes The app is now being used in the research department at St Thomas' to calculate accurate centiles for research participants at the Centre for the Developing Brain. Despite the deliberately local launch of the first version, the app has already been downloaded by 2,500 users. It is hoped that as the app gains adoption, the calculation of growth centiles in paediatric practice will become more widespread, with associated benefits to child health (Figure 1).

Lessons learnt I have learned about implementing a new tool in clinical practice, combined with gathering feedback and suggestions from a wide range of people to make the product as clinically useful as possible.

Message for others Creating a web/app-based tool for commonly-performed error-prone tasks appears to be an effective strategy to improve quality at the bedside. There are many resources available online to guide interested people through this process. 
G574(P) IMPROVING PATIENT CARE THROUGH PAEDIATRIC SIMULATION AND MULTI-DISCIPLINARY RESUSCITATION TRAINING BASED UPON PREVIOUS SERIOUS INCIDENTS AT A DISTRICT GENERAL HOSPITAL

J Halbert, M Virgilio, A Dhelaria. Paediatrics, Lister Hospital, Stevenage, UK

\subsection{6/archdischild-2015-308599.523}

Context The quality improvement project was undertaken at a District General Hospital and included staff who care for seriously sick or injured children including doctors and nurses working in general paediatrics, accident and emergency and anaesthetics. The appropriate initial assessment and resuscitation of serious ill and injured children together with on-going reassessment and emergency management are essential for reducing morbidity and mortality.

Problem There was no integrated multi-disciplinary team (MDT) paediatric resuscitation training and several paediatric serious incidents had involved more than one department. Simulation training increases the acquisition of skills and provides a platform to review performance and make errors without compromising patient safety. Both the Department of Health and $\mathrm{RCPCH}$ recognise the importance of simulation training in improving patient safety through reducing human and system errors. High fidelity manikins can be monitored which significantly adds to their realism.

Assessment of problem and analysis of its causes A retrospective review was undertaken by a paediatric trainee of all the paediatric serious incidents during a ten year period to identify common themes and key recommendations. The development of MDT resuscitation training was one of the principle recommendations to improve the emergency assessment and care provided to children.

Intervention MDT paediatric resuscitation training sessions involving healthcare assistants, nurses, doctors and other allied healthcare professionals from general paediatrics, accident and emergency and anaesthetics were planned several months in advance through a consultation process. Dates and times were chosen to maximise trainee availability and minimise disruption to routine services. For MDT training in accident and emergency, strategies were implemented to ensure patient safety at all times. Resuscitation officers provided support and resuscitation equipment. The hospital switch board was notified so a simulated paediatric crash call could be initiated through the hospital paging system. Scenarios were developed based upon previous paediatric serious incidents to review whether key recommendations had been successfully implemented and sustained. The focus was on familiarisation with paediatric resuscitation equipment, drugs and guidelines in real time as well as teamwork, communication and leadership skills and dynamics between and within the different specialties. A pilot paediatric simulation MDT training session was also undertaken in the Education Centre.

Strategy for change MDT paediatric resuscitation training was coordinated through the paediatric and emergency departments as well as the hospital resuscitation committee. Anonymous feedback through questionnaires was collected from participants.

Measurement of improvement The MDT paediatric resuscitation and simulation training received very positive feedback: "Excellent scenario, felt more real than other simulated scenarios I have attended." Participants found the MDT simulation training much more realistic than weekly departmental resuscitation training with $79 \%$ strongly recommending the simulation training compared with just $33 \%$ for the weekly departmental training.
Effects of changes MDT paediatric resuscitation training was successfully implemented. A business case was developed for the purchase of a paediatric simulation manikin to improve the reality of the training.

Lessons learnt MDT paediatric resuscitation training is a very valuable tool for improving teamwork and paediatric resuscitation skills. Paediatric high fidelity simulation training can enhance this further through providing a more realistic experience.

Message for others MDT resuscitation training should be routine practice in district general hospitals. Basing scenarios on previous serious incidents is a useful way to review previous key recommendations. A business case can be developed by demonstrating improved experience through a pilot high fidelity simulation trainer.

\section{G575(P) PROMOTING QUALITY IN A COMMUNITY PAEDIATRIC DEPARTMENT}

C Furnell, F Finlay. Child Health, Sirona Care and Health, Bath, UK

\subsection{6/archdischild-2015-308599.524}

Context Work was done within a busy community paediatric department. The work looked at how we assess and promote quality.

Problem A lack of focus and evidence of quality.

Assessment of problem and analysis of its causes Within the department we had completed audits over the years on an adhoc basis, there were guidelines and pathways which were used but they were not always up to date. There was no universal way documentation could be accessed to see what had been done when, or when it needed repeating/updating. This meant that there was variability in the service we provided.

Intervention Initially we spent 2 days collecting data about the quality work being done already. Next we planned four interventions.

1. We created a database of audits, who was responsible for them and when they needed repeating. The database also noted if there were protocols and audit tools and if the audit had been presented.

2. We formed a centralised set of online files based on the current CQC standards. Supporting evidence was filed making it accessible to everyone in the department. Audit reports, pathways, patient information sheets, induction material and department protocols can be easily found.

3. We asked each member of the department to join a "champions" group focused on different areas of change. New trainees in the department are allocated to a group. These included:-

- Staff Wellbeing

- ASD

- ADHD

- Patient Voice

- Expectations

1. We organised a monthly "quality" meeting. This is an opportunity for the department to present any work so that we can all learn and share. Changes can be ratified quickly so consistent high quality care can be provided.

Strategy for change The most important strategy was encouraging each team member to feel responsibilty for quality. An away day allowed us to develop a shared vision and the champion 